Am J Sports Med. 2017 September ; 45(11): 2586-2594. doi:10.1177/0363546517712952.

\title{
Surgical Predictors of Clinical Outcome following Revision ACL Reconstruction
}

\author{
A full list of authors and affiliations appears at the end of the article.
}

\begin{abstract}
Background-Revision anterior cruciate ligament (ACL) reconstruction has been documented to have worse outcomes compared with primary ACL reconstructions. The reasons why remain varied. The purpose of this study was to determine whether previous or current surgical factors noted at the time of revision ACL reconstruction are significant predictors towards activity level, sports function, and osteoarthritis (OA) symptoms at 2-year follow-up.
\end{abstract}

Hypothesis-Certain factors under the control of the surgeonat the time of revision surgery can both negatively and positively impact outcome.

Study Design—Cohort Study; Level of evidence, 2.

Methods-Revision ACL reconstruction patients were identified and prospectively enrolled between 2006 and 2011. Data collected included baseline demographics, intra-operative surgical technique and joint pathology, and a series of validated patient-reported outcome instruments (International Knee Documentation Committee [IKDC], Knee Injury and Osteoarthritis Outcome Score [KOOS], Western Ontario McMaster Universities Osteoarthritis Index [WOMAC], and Marx activity rating score) completed prior to surgery. Patients were followed up for 2 years, and asked to complete the identical set of outcome instruments.

Regression analysis was used to control for age, gender, body mass index (BMI), activity level, baseline outcome scores, revision number, time since last ACL reconstruction, and a variety of previous and current surgical variables, in order to assess the surgical risk factors for clinical outcomes 2 years after revision ACL reconstruction.

Results-A total of 1205 patients (697 [58\%] males) met the inclusion criteria and were successfully enrolled. The median age was 26 years, and median time since their last ACL reconstruction was 3.4 years.

Two-year follow-up was obtained on 82\% (989/1205). Both previous as well as current surgical factors were found to be significant contributors towards poorer clinical outcomes at 2 years. The most consistent surgical factors driving outcome in revision patients were prior surgical approach (arthrotomy vs. no arthrotomy), prior tibial tunnel position, femoral fixation at the time of revision, and having a notchplasty. Having a previous arthrotomy (non-arthroscopic open approach) for ACL reconstruction compared to the one-incision technique resulted in significantly

Corresponding Author: Rick W. Wright, MD, Washington University St. Louis, 660 S. Euclid Ave, Campus Box 8233, St. Louis, MO 63110, rwwright1@aol.com.

Barton Mann, PhD (deceased)

Timothy M. Hosea, MD (deceased) 
poorer outcomes on 2-year IKDC ( $\mathrm{p}=0.037$; odds ratio[OR $]=2.43$; 95\% CI, 1.05-5.88) and KOOS pain, sports/rec, and quality of life (QOL) subscales ( $\mathrm{p} \unlhd$ ).05; OR range $=2.38-4.35$; 95\% CI, $1.03-$ 10.0). Using a metal interference screw for current femoral fixation resulted in significantly better outcomes in 2-year KOOS symptoms, pain, and QOL subscales ( $\mathrm{p} \unlhd 0.05$; OR range $=1.70-1.96$; 95\% CI, 1.00-3.33), as well as WOMAC stiffness ( $\mathrm{p}=0.041$; OR=1.75; 95\% CI, 1.02-3.03). Not having a notchplasty at revision significantly improved 2-year outcomes of the IKDC ( $\mathrm{p}=0.013$; $\mathrm{OR}=1.47$; 95\% CI, 1.08-1.99), KOOS activities of daily living (ADL) and QOL subscales (p $\unlhd$ ).04; OR range $=1.40-1.41 ; 95 \% \mathrm{CI}, 1.03-1.93$ ), and the WOMAC stiffness and ADL subscales (p $₫ .04$; OR range $=1.41-1.49 ; 95 \% \mathrm{CI}, 1.03-2.05$ ).

Factors prior to revision ACL that increase risk of poorer clinical outcomes at two years include lower baseline outcome scores, lower Marx activity score at the time of revision, higher BMI, female gender, and shorter time since the patient's last ACL reconstruction.

Prior femoral fixation, prior femoral aperture position, and the knee flexion angle at the time of revision graft fixation were not found to affect2-year outcomes in this revision cohort.

Conclusions-There are certain surgical variables the physician can control at the time of an ACL revision that can modify clinical outcomes at 2 years. Whenever possible, opting for an anteromedial portal or transtibial surgical exposure, choosing a metal interference screw for femoral fixation, and not having a notchplasty are associated with a significantly better 2-year clinical outcome.

Clinical Relevance-Revision ACL reconstruction remains a challenging clinical situation with revisions resulting in worse outcomes than primary ACL reconstructions. This study adds to the growing body of evidence to improve revision results. Some surgical variables may be utilized to help improve outcome.

\section{Keywords}

anterior cruciate ligament; revision ACL reconstruction; outcomes; surgical factors; surgical approach; tunnel position; ACL fixation

\section{What is known about the subject}

Little was known prior to the analysis of this cohort regarding surgical options impacting outcome. Most previous studies have centered upon failure, patient-reported outcomes and graft choice surrounding revision reconstruction and have not had the ability to assess the impact of surgical options due to the relatively small clinical series.

\section{Adds to existing knowledge}

This study provides evidence from a large prospective ACL revision cohort that surgical factors can be significant contributors towards poorer clinical outcomes at 2 years.

\section{INTRODUCTION}

Revision anterior cruciate ligament (ACL) reconstruction has been documented to have worse outcomes compared with primary ACL reconstructions. ${ }^{1-3,8-10,15,20,22,23,25,26}$ The 
Multicenter ACL Revision (MARS) group has identified several contributing factors for outcomes, including graft choice, previous lateral meniscectomy, and trochlear groove chondrosis. ${ }^{11,12}$ Other factors remain unknown. Numerous factors remain beyond the control of the patient or the surgeon with regards to revision ACL reconstructions. Fortunately, some factors can be chosen by the surgeon when planning reconstruction.

ACL graft choice at the time of revision reconstruction has been shown to affect outcome. ${ }^{5,12,14}$ In a previous study by the MARS group it was demonstrated that the use of an autograft (compared to an allograft) is associated with an improved return to sports and decreased risk of graft re-rupture by 2.78 times. ${ }^{12}$ Additional factors such as surgical approach (e.g., anteromedial portal, transtibial, 2 incision, arthrotomy), tunnel choice (new, old or "blended", defined as the combination of old and new tunnels), bone grafting, and fixation choice may have the ability to offer options for the operating surgeon. The purpose of this study was to determine if either previous or current surgical factors noted at the time of ACL revision reconstruction predicted activity level, sports function, and osteoarthritis symptoms at 2-year follow-up. Our hypothesis is that surgical factors under the control of the surgeon (e.g., surgical approach, tunnel choice, notchplasty, bone grafting, fixation choice) can both negatively and positively impact revision ACL reconstruction outcome.

\section{METHODS}

\section{Setting and Study Population}

The MARS Group was assembled with the aim of determining what impacts outcome in an ACL revision setting, and to identify potentially modifiable factors that could improve these outcomes. ${ }^{6,13,24,27}$ This collaboration consists of a group of 83 sports medicine fellowship trained surgeons across 52 sites. Surgeons are a near equal mix of academic and private practitioners. After obtaining approval from respective institutional review boards (IRBs), this multicenter consortium began patient enrollment in 2006 and ended in 2011, during which time 1205 revision ACL reconstruction patients were enrolled in this prospective longitudinal cohort. The study enrolled patients undergoing revision of a previously failed ACL reconstruction (as identified by clinical exam, imaging, or arthroscopic confirmation) who agreed to participate, signed an informed consent, and completed a series of patientreported outcome instruments. Indications for the revision ACL reconstruction included functional instability, abnormal laxity testing or an MRI indicating graft tear. Multi-ligament reconstructions were excluded. Ligament injuries not requiring reconstruction (i.e., MCL) were included. Surgeon inclusion criteria included maintenance of an active IRB approval, completion of a training session that integrated articular cartilage and meniscus agreement studies, review of the study design and patient inclusion criteria, and a review of the surgeon questionnaire. ${ }^{18}$ Surgical technique was at the discretion of the treating surgeon.

\section{Data Sources and Measurement}

After obtaining informed consent, the patient filled out a 13-page questionnaire that included questions regarding demographics, sports participation, injury mechanism, comorbidities and knee injury history, as previously described. ${ }^{12,13}$ Within this questionnaire, each participant also completed a series of validated general and knee-specific outcome 
instruments, including the Knee Injury and Osteoarthritis Outcome Score (KOOS), the International Knee Documentation Committee Subjective form (IKDC) and the Marx activity rating scale. Contained within the KOOS was the Western Ontario and McMaster Universities Osteoarthritis Index (WOMAC). Surgeons filled out a 42-page questionnaire that included the impression of the etiology of the previous failure, physical exam findings, surgical technique utilized, the intra-articular findings and surgical management of meniscal and chondral damage.

Completed data forms were mailed from each participating site to the data coordinating center. Data from both the patient and surgeon questionnaires were scanned with Teleform ${ }^{\mathrm{TM}}$ software (Cardiff Software, Inc., Vista, CA) utilizing optical character recognition, and the scanned data was verified and exported to a master database. A series of logical error and quality control checks were subsequently performed prior to data analysis.

\section{Patient Follow-up}

Two-year patient follow-up was completed by mail with re-administration of the same questionnaire as the one they completed at baseline. Patients were also contacted by phone to determine whether any subsequent surgery had occurred to either knee since their initial revision ACL reconstruction. If so, operative reports were obtained, whenever possible, in order to verify pathology and treatment.

\section{Statistical Analysis}

To describe our patient sample, we summarized continuous variables as percentiles (i.e., 25th, 50th, and 75th), and categorical variables with frequencies and percentages. Multivariable regression analyses were constructed to examine which baseline risk factors were independently associated with each outcome variable. The primary outcome variables of interest were the 2-year outcome scores of the KOOS, IKDC, WOMAC and Marx activity level. These primary outcome variables were all treated as continuous, and as such, ordinal logistic regression models were used. All models controlled for age, gender, body mass index (BMI), activity level, baseline outcome scores, revision number, time from previous ACL reconstruction, and a variety of previous and current surgical variables (including graft choice, meniscal and chondral damage), in order to assess the surgical risk factors for clinical outcomes 2 years after revision surgery. Per number of levels, categorical variables were fit according to their degrees of freedom (i.e. n-1). To stay within the allowable degrees of freedom, each continuous variable was fit as a linear effect, as there was little or no evidence of a non-linear relationship with a p-value $\leq 0.05$ for the non-linear test. Statistical analysis was performed using open source R statistical software (www.r-project.org; Version 3.0.3).

\section{RESULTS}

\section{Study Population and Follow-up}

A total of 1205 patients (697 [58\%] males) met the inclusion criteria and were successfully enrolled. The median age was 26 years, and median time since the patients' last ACL 
reconstruction was 3.4 years. Baseline characteristics of the cohort are summarized in Table 1. At 2 years, questionnaire follow-up was obtained on $82 \%$ (989/1205).

\section{Influence of Surgical Factors on 2-Year Outcomes}

A variety of surgeon-based surgical factors predicted outcome. Both previous as well as current surgical factors were found to be associated with poorer outcomes at 2 years (Table 2).

A. Surgical Approach and Tunnel Choice-A history of arthrotomy at the time of the previous reconstruction (compared to a one-incision technique) was associated with significantly poorer outcomes on 2-year IKDC ( $\mathrm{p}=0.037$; odds ratio $[\mathrm{OR}]=2.43 ; 95 \% \mathrm{CI}$, 1.05-5.88) and KOOS pain, sports/recreation, and quality of life (QOL) subscales ( $\mathrm{p} \unlhd$ 0.05; OR range $=2.38-4.35 ; 95 \% \mathrm{CI}, 1.03-10.0$ ). In particular, patients having a previous arthrotomy from their previous reconstruction were 4.35 times more likely to have a poorer KOOS QOL outcome at 2 years, compared with a patient who had a previous one-incision approach $(\mathrm{p}=0.001$ ). Patients having a history of double femoral tunnels were 3.13 times more likely to have a poorer KOOS QOL outcome at 2 years, compared with patients who had a single femoral tunnel $(\mathrm{p}=0.027)$. A prior tibial tunnel aperture position defined as 'ideal' in position and size by the participating MARS surgeon at the time of the revision surgery was associated with significantly worse 2-year clinical outcomes in nearly all instruments (IKDC; KOOS symptoms, pain, ADL, sports/rec, QOL subscales; WOMAC stiffness, pain, ADL subscales), when compared to a tibial aperture position of "ideal in both position and size, but enlarged tunnels".

At revision surgical exposure with a two-incision technique had worse Marx $(\mathrm{p}=0.029)$ and KOOS symptoms ( $\mathrm{p}=0.028$ ) scores compared with anteromedial portal femoral tunnel drilling. Transtibial vs. anteromedial approach was not associated with outcome. Choosing to utilize a previous femoral tunnel that was deemed to be in the optimum position versus drilling an entirely new tunnel was associated with worse KOOS QOL scores $(\mathrm{p}=0.025)$.

Choosing to drill a second tibial tunnel versus utilizing the previous tibial tunnel position was associated with a significantly worse KOOS ADL and WOMAC ADL outcome scores at 2 years $(\mathrm{p}=0.026)$. In particular, a patient needing a $2^{\text {nd }}$ tibial tunnel drilled had a 3.45 times higher likelihood of having a poorer 2-year KOOS ADL and WOMAC ADL score, when compared to the tibial tunnel being in the optimum position at the time of the revision surgery.

Patients who had a notchplasty at the time of revision had worse IKDC, KOOS ADL and QOL, and WOMAC stiffness and ADL scores. Revisions without a notchplasty had significantly improved 2-year outcomes of the IKDC ( $\mathrm{p}=0.013$; OR=1.47; 95\% CI, 1.081.99), KOOS ADL and QOL subscales (p $₫$ ).04; OR range $=1.40-1.41$; 95\% CI, 1.03-1.93), and the WOMAC stiffness and ADL subscales ( $\mathrm{p} \unlhd$ (0.04; OR range $=1.41-1.49 ; 95 \% \mathrm{CI}$, $1.03-2.05)$.

B. Fixation Choice-Using a metal interference screw for current revision femoral fixation (compared with bioabsorbable interference screws, cross pins, or a combination of 
fixation devices) was associated with significantly better outcomes in 2-year KOOS symptoms, pain, and QOL subscales ( $₫ \unlhd .05$; OR range $=1.70-1.96$; 95\% CI, 1.00-3.33), as well as WOMAC stiffness ( $\mathrm{p}=0.041$; $\mathrm{OR}=1.75 ; 95 \% \mathrm{CI}, 1.02-3.03$ ). Similarly, using a metal interference screw for current revision tibial fixation (compared with using a combination of fixation devices) was associated with significantly better IKDC ( $\mathrm{p}=0.017)$ and WOMAC stiffness $(\mathrm{p}=0.013)$ scores.

C. Biology-Femoral tunnel bone grafting, either single or two staged, was associated with worse Marx scores at 2 years ( $\mathrm{p}=0.048$; OR=2.04; 95\% CI, 1.00-4.17). Conversely, patients who required tibial tunnel bone grafting (single or two staged) actually reported improved outcomes for KOOS pain ( $\mathrm{p}=0.046$ ) and WOMAC pain $(\mathrm{p}=0.004)$. Utilization of a biologic enhancement agent (i.e. platelet rich plasma, mesenchymal stem cells) was associated with worse Marx activity level scores at 2 years $(\mathrm{p}=0.025)$.

In summary, the most consistent surgical factors associated with better outcome in revision patients were prior surgical approach, prior tibial tunnel position, current femoral fixation, and not having a notchplasty. Conversely, prior femoral fixation, prior femoral aperture position, and the knee flexion angle at the time of graft fixation were not found to be associated with2-year outcomes in this revision cohort.

\section{Influence of Patient Characteristics on 2-Year Outcomes}

Lower baseline outcome scores predicted worse 2-year outcomes for Marx activity, all KOOS subscales, IKDC, and all WOMAC subscales ( $\mathrm{p}<0.001)$. (Table 2) Lower baseline Marx activity scores predicted worse 2-year Marx activity, KOOS pain, ADL, sports/ recreation, QOL, WOMAC pain and ADL subscales $(\mathrm{p}<0.01)$. Higher BMI predicted worse outcomes for all KOOS subscales, the IKDC and WOMAC pain and ADL subscales $(\mathrm{p}<0.01)$. Female gender predicted worse outcome for Marx, KOOS ADLs, IKDC, WOMAC pain and ADL subscales. Age (increased) predicted lower 2-year Marx activity level scores $(\mathrm{p}<0.001)$. A shorter time since the last ACL reconstruction predicted worse outcomes for all $5 \mathrm{KOOS}$ subscales and all WOMAC subscales in addition to the IKDC ( $\mathrm{p} \unlhd$ (002). A second revision or higher predicted a worse outcome for KOOS knee-related $\mathrm{QOL}(\mathrm{p}=0.014)$. If the surgeon was revising a patient they had not previously reconstructed it predicted a worse Marx score at 2 years $(\mathrm{p}=0.015)$.

\section{DISCUSSION}

The goal of this study was to determine if surgeon modifiable factors could be identified that are associated with improved outcome. While there are a few findings that can be impacted by the surgeon, many are beyond the control or do not impact outcome enough to drive technique changes. Tunnel position, fixation, bone grafting and biologic agent usage are at least somewhat controlled by the surgeon and are associated with outcome.

Tunnel position has a variety of presentations in the revision setting and how to drill the new tunnel may be controllable for the surgeon. The pre-existing tunnel may be appropriately placed and utilized again, it may be so poorly positioned that an entirely new tunnel is drilled or it may be a combination which when drilled again results in a blended (blended $=$ 
a combination old and new tunnel) tunnel that may have a wider aperture. It was feared that a blended tunnel with a wide aperture might result in worse outcomes or higher failure rates. Interestingly, a blended tunnel for the femur and tibia did not impact outcome. However, utilizing a previous tunnel did not result in outcomes as good as those obtained by a completely new tunnel. It may be surmised that at times using a previous tunnel was at some level a compromise of position, by not wanting a blended tunnel. Additionally, revision graft healing within a previously utilized tunnel may impact outcome at a level this current study is unable to detect or measure. There may be biological factors we are yet able to detect that compromise outcome despite correctly drilled tunnels and appropriately placed grafts.

Additionally, some factors that predict outcome in this study may not actually be causative, but are surrogates for factors we have not yet identified with our research.

Transtibial drilling did not predict outcome despite some surgeons' belief that anteromedial portal drilling allows independent and improved ability to localize the femoral tunnel.

Previous clinical studies have corroborated this finding that anteromedial portal drilling while theoretically an improvement has not necessarily been verified in clinical findings in the primary ACL reconstruction setting. ${ }^{19,21}$ Two-incision femoral tunnel drilling versus anteromedial drilling impacted outcome as measured by the KOOS Symptoms subscale $(\mathrm{p}=0.028, \mathrm{OR}=1.52)$. A previous study has not corroborated this finding where both methods resulted in similar outcomes. ${ }^{16}$

Graft fixation surprisingly impacted outcome in this revision setting. Fixation has rarely been demonstrated to make a clinical difference in the primary setting, where most fixation methods appear adequate for both soft tissue autografts and allografts and patellar tendon autografts and allografts. ${ }^{4,7,17}$ In the current study, metal femoral fixation resulted in significantly improved KOOS pain, symptoms and QOL subscales. Additionally, use of a metal screw versus a combination of fixation for the tibia improved IKDC and WOMAC stiffness scores. It is not possible to determine the exact pathophysiological reason that this predicts outcome, but bone quality is often worse in the revision setting due to previous tunnels even if not enlarged and use of a metal fixation may overcome some of this challenge. Additionally, metal as an inert implant may offer less reactivity than bioabsorbable in the revision ACL reconstruction setting.

Bone grafting either single or two staged of dilated tunnels can be challenging for patients, resulting in additional surgery and time to ultimate revision if staged. Thus, it is important to determine if this impacts outcome. For dilated tibial tunnels requiring bone grafting it significantly improves patient outcomes as measured by KOOS and WOMAC pain scores. Unfortunately, femoral tunnel bone grafting predicted a worse Marx activity score at 2 years. This represents one of those findings that are challenging to incorporate in practice. Bone grafting a femoral tunnel too dilated should not be avoided to try to improve 2-year Marx scores. Also, utilization of biologic agents to enhance surgical results was not shown to improve outcome and in fact demonstrated worse 2-year MARX scores.

Other factors that were noted to impact outcome, but may not be modifiable include performance of a notchplasty, which resulted in worse KOOS ADL and QOL, IKDC and WOMAC stiffness and ADL scores. If a notchplasty is definitely needed as determined by 
the surgeon then there remains little choice in performing this step in reconstruction. Typically, in the revision setting this represents notch overgrowth and may be a surrogate indicator of degenerative processes occurring throughout the joint. Within the limits of our study it remains uncertain why a notchplasty would be associated with worse outcome, but our analysis technique controls for a variety of variables including chondral damage and thus it remains an independent predictor. Presence or absence of notchplasty is all that is recorded so size or amount of notchplasty may matter, but that is beyond the scope of our study. The presence of two femoral tunnels from previous surgery is associated with a worse outcome, but is not a surgically modifiable variable. A previous arthrotomy resulted in worse outcome, but is also not able to be modified.

Strengths of the study include the prospective data collection of validated patient-reported outcome measures with the largest prospective revision ACL reconstruction cohort collected to date. This allows multivariable analysis of a high number of factors. Weaknesses include no onsite follow-up, surgeon variation in tunnel drilling as to blended vs. previous tunnel usage, and inability to control indications for bone grafting, tunnel placement and fixation choice by surgeons.

\section{CONCLUSIONS}

A variety of surgical variables are represented in the revision ACL reconstruction setting. Some are modifiable, but unfortunately many remain beyond the individual surgeon's control. The strongest predictor for revision surgery that is controlled by the surgeon is femoral fixation where a metal screw improved outcome. Additional factors that less strongly impacted outcome included drilling a new femoral tunnel vs. utilizing a previous tunnel, and bone grafting the tibia when indicated. Surgical approach for femoral drilling was not a large factor with no advantage of anteromedial versus transtibial, but some improvement of anteromedial over two-incision. Surgeons must balance a variety of these factors in revision ACL reconstruction outcomes along with graft choice, meniscal and articular cartilage findings and management to optimize outcome in these challenging clinical settings.

\section{Authors}

The MARS Group, Christina R. Allen, MD, University of California, San Francisco, San Francisco, California USA

Allen F. Anderson, MD, Tennessee Orthopaedic Alliance, Nashville, TN USA

Daniel E. Cooper, MD, W.B. Carrell Memorial Clinic, Dallas, TX USA

Thomas M. DeBerardino, MD, The San Antonio Orthopaedic Group, San Antonio, TX USA

Warren R. Dunn, MD, MPH, Reedsburg Area Medical Center, Reedsburg, WI USA 
Amanda K. Haas, MA,

Washington University in St. Louis, St. Louis, MO USA

Laura J. Huston, MS,

Vanderbilt University, Nashville, TN USA

Brett (Brick) A. Lantz, MD,

Slocum Research and Education Foundation, Eugene, OR USA

Barton Mann, PhD,

AOSSM, Rosemont, IL USA

Sam K Nwosu, MS,

Vanderbilt University, Nashville, TN, USA

Kurt P. Spindler, MD,

Cleveland Clinic, Cleveland, OH USA

Michael J. Stuart, MD,

Mayo Clinic, Rochester, MN USA

Rick W. Wright, MD,

Washington University in St. Louis, St. Louis, MO USA

John P. Albright, MD,

University of lowa Hospitals and Clinics, lowa City, IA USA

Annunziato (Ned) Amendola, MD,

Duke University, Durham, NC USA

Jack T. Andrish, MD,

Cleveland Clinic, Cleveland, OH USA

Christopher C. Annunziata, MD,

Commonwealth Orthopaedics \& Rehabilitation, Arlington, VA USA

Robert A. Arciero, MD,

University of Connecticut Health Center, Farmington, CT USA

Bernard R. Bach Jr, MD,

Rush University Medical Center, Chicago, IL USA

Champ L. Baker III, MD,

The Hughston Clinic, Columbus, GA USA

Arthur R. Bartolozzi, MD,

3B Orthopaedics, University of Pennsylvania Health System, Philadelphia, PA USA

Keith M. Baumgarten, MD,

Orthopedic Institute, Sioux Falls, SD USA

Jeffery R. Bechler, MD,

University Orthopaedic Associates LLC, Princeton, NJ USA

Jeffrey H. Berg, MD, 
own Center Orthopaedic Associates, Reston, VA USA

Geoffrey A. Bernas, MD,

State University of New York at Buffalo, Buffalo, NY

Stephen F. Brockmeier, MD,

University of Virginia, Charlottesville, VA USA

Robert H. Brophy, MD,

Washington University in St. Louis, St. Louis, MO USA

Charles A. Bush-Joseph, MD,

Rush University Medical Center, Chicago, IL USA

J. Brad Butler V, MD,

Orthopedic and Fracture Clinic, Portland, OR USA

John D. Campbell, MD,

Bridger Orthopedic and Sports Medicine, Bozeman, MT USA

James L. Carey, MD, MPH,

University of Pennsylvania, Philadelphia, PA USA

James E. Carpenter, MD,

University of Michigan, Ann Arbor, MI USA

Brian J. Cole, MD,

Rush University Medical Center, Chicago, IL USA

Jonathan M. Cooper, DO,

HealthPartners Specialty Center, St. Paul, MN USA

Charles L. Cox, MD, MPH,

Vanderbilt University, Nashville, TN USA

R. Alexander Creighton, MD,

University of North Carolina Medical Center, Chapel Hill, NC USA

Diane L. Dahm, MD,

Mayo Clinic, Rochester, MN USA

Tal S. David, MD,

Synergy Specialists Medical Group, San Diego, CA USA

David C. Flanigan, MD,

The Ohio State University, Columbus, OH USA

Robert W. Frederick, MD, The Rothman Institute/Thomas Jefferson University, Philadelphia, PA USA

Theodore J. Ganley, MD,

Children's Hospital of Philadelphia, Philadelphia, PA USA

Elizabeth A. Garofoli,

Washington University in St. Louis, St. Louis, MO USA 
Charles J. Gatt Jr, MD,

University Orthopaedic Associates LLC, Princeton, NJ USA

Steven R. Gecha, MD,

Princeton Orthopaedic Associates, Princeton, NJ USA

James Robert Giffin, MD,

Fowler Kennedy Sport Medicine Clinic, University of Western Ontario, London

Ontario, Canada

Sharon L. Hame, MD,

David Geffen School of Medicine at UCLA, Los Angeles, CA USA

Jo A. Hannafin, MD, PhD,

Hospital for Special Surgery, New York, NY USA

Christopher D. Harner, MD,

University of Texas Health Center, Houston, TX USA

Norman Lindsay Harris Jr, MD,

Grand River Health in Rifle, CO USA

Keith S. Hechtman, MD,

UHZ Sports Medicine Institute, Coral Gables, FL USA

Elliott B. Hershman, MD,

Lenox Hill Hospital, New York, NY USA

Rudolf G. Hoellrich, MD,

Slocum Research and Education Foundation, Eugene, OR USA

Timothy M. Hosea, MD,

University Orthopaedic Associates LLC, Princeton, NJ USA

David C. Johnson, MD,

National Sports Medicine Institute, Leesburg, VA USA

Timothy S. Johnson, MD,

National Sports Medicine Institute, Leesburg, VA USA

Morgan $\mathrm{H}$. Jones, MD,

Cleveland Clinic, Cleveland, OH USA

Christopher C. Kaeding, MD,

The Ohio State University, Columbus, OH USA

Ganesh V. Kamath, MD,

University of North Carolina Medical Center, Chapel Hill, NC USA

Thomas E. Klootwyk, MD,

Methodist Sports Medicine, Indianapolis, IN USA

Bruce A. Levy, MD,

Mayo Clinic Rochester, MN USA 
C. Benjamin Ma, MD, University of California, San Francisco, CA USA

G. Peter Maiers II, MD, Methodist Sports Medicine Center, Indianapolis, IN USA

Robert G. Marx, MD, Hospital for Special Surgery, New York, NY USA

Matthew J. Matava, MD, Washington University in St. Louis, St. Louis, MO USA

Gregory M. Mathien, MD, Knoxville Orthopaedic Clinic, Knoxville, TN USA

David R. McAllister, MD, David Geffen School of Medicine at UCLA, Los Angeles, CA USA

Eric C. McCarty, MD, University of Colorado Denver School of Medicine, Denver, CO USA

Robert G. McCormack, MD, University of British Columbia, New Westminster, BC Canada

Bruce S. Miller, MD, MS, University of Michigan, Ann Arbor, MI USA

Carl W. Nissen, MD,

Connecticut Children's Medical Center, Hartford, CT USA

Daniel F. O'Neill, MD, EdD,

Littleton Regional Healthcare, Littleton, NH USA

Brett D. Owens, MD,

Warren Alpert Medical School, Brown University, Providence, RI USA

Richard D. Parker, MD,

Cleveland Clinic, Cleveland, OH USA

Mark L. Purnell, MD, Orthopaedic Associates of Aspen \& Glenwood, Aspen, CO USA

Arun J. Ramappa, MD, Beth Israel Deaconess Medical Center, Boston, MA USA

Michael A. Rauh, MD, State University of New York at Buffalo, Buffalo, NY USA

Arthur C. Rettig, MD, Methodist Sports Medicine, Indianapolis, IN USA

Jon K. Sekiya, MD, University of Michigan, Ann Arbor, MI USA

Kevin G. Shea, MD, 
Intermountain Orthopaedics, Boise, ID USA

Orrin H. Sherman, MD,

NYU Hospital for Joint Diseases, New York, NY USA

James R. Slauterbeck, MD,

Robert Larner College of Medicine, University of Vermont, Burlington, VT USA

Matthew V. Smith, MD,

Washington University in St. Louis, St. Louis, MO USA

Jeffrey T. Spang, MD,

University of North Carolina Medical Center, Chapel Hill, NC USA

LTC Steven J. Svoboda, MD,

Keller Army Community Hospital, United States Military Academy, West Point, NY USA

Timothy N. Taft, MD,

University of North Carolina Medical Center, Chapel Hill, NC USA

Joachim J. Tenuta, MD,

Albany Medical Center, Albany, NY USA

Edwin M. Tingstad, MD,

Inland Orthopaedic Surgery and Sports Medicine Clinic, Pullman, WA USA

Armando F. Vidal, MD,

University of Colorado Denver School of Medicine, Denver, CO USA

Darius G. Viskontas, MD,

Royal Columbian Hospital, New Westminster, BC Canada

Richard A. White, MD,

St. Mary's Audrain, Mexico, MO USA

James S. Williams Jr, MD,

Cleveland Clinic, Euclid, OH USA

Michelle L. Wolcott, MD,

University of Colorado Denver School of Medicine, Denver, CO USA

Brian R. Wolf, MD, and

University of lowa Hospitals and Clinics, lowa City, IA USA

James J. York, MD

\section{Affiliations}

University of California, San Francisco, San Francisco, California USA

Tennessee Orthopaedic Alliance, Nashville, TN USA

W.B. Carrell Memorial Clinic, Dallas, TX USA

The San Antonio Orthopaedic Group, San Antonio, TX USA 
Reedsburg Area Medical Center, Reedsburg, WI USA

Washington University in St. Louis, St. Louis, MO USA

Vanderbilt University, Nashville, TN USA

Slocum Research and Education Foundation, Eugene, OR USA

AOSSM, Rosemont, IL USA

Vanderbilt University, Nashville, TN, USA

Cleveland Clinic, Cleveland, OH USA

Mayo Clinic, Rochester, MN USA

Washington University in St. Louis, St. Louis, MO USA

University of lowa Hospitals and Clinics, lowa City, IA USA

Duke University, Durham, NC USA

Cleveland Clinic, Cleveland, OH USA

Commonwealth Orthopaedics \& Rehabilitation, Arlington, VA USA

University of Connecticut Health Center, Farmington, CT USA

Rush University Medical Center, Chicago, IL USA

The Hughston Clinic, Columbus, GA USA

3B Orthopaedics, University of Pennsylvania Health System, Philadelphia, PA USA

Orthopedic Institute, Sioux Falls, SD USA

University Orthopaedic Associates LLC, Princeton, NJ USA

own Center Orthopaedic Associates, Reston, VA USA

State University of New York at Buffalo, Buffalo, NY

University of Virginia, Charlottesville, VA USA

Washington University in St. Louis, St. Louis, MO USA

Rush University Medical Center, Chicago, IL USA

Orthopedic and Fracture Clinic, Portland, OR USA

Bridger Orthopedic and Sports Medicine, Bozeman, MT USA

University of Pennsylvania, Philadelphia, PA USA

University of Michigan, Ann Arbor, MI USA

Rush University Medical Center, Chicago, IL USA

HealthPartners Specialty Center, St. Paul, MN USA

Vanderbilt University, Nashville, TN USA

University of North Carolina Medical Center, Chapel Hill, NC USA 
Mayo Clinic, Rochester, MN USA

Synergy Specialists Medical Group, San Diego, CA USA

The Ohio State University, Columbus, $\mathrm{OH}$ USA

The Rothman Institute/Thomas Jefferson University, Philadelphia, PA USA

Children's Hospital of Philadelphia, Philadelphia, PA USA

Washington University in St. Louis, St. Louis, MO USA

University Orthopaedic Associates LLC, Princeton, NJ USA

Princeton Orthopaedic Associates, Princeton, NJ USA

Fowler Kennedy Sport Medicine Clinic, University of Western Ontario, London Ontario, Canada

David Geffen School of Medicine at UCLA, Los Angeles, CA USA

Hospital for Special Surgery, New York, NY USA

University of Texas Health Center, Houston, TX USA

Grand River Health in Rifle, CO USA

UHZ Sports Medicine Institute, Coral Gables, FL USA

Lenox Hill Hospital, New York, NY USA

Slocum Research and Education Foundation, Eugene, OR USA

University Orthopaedic Associates LLC, Princeton, NJ USA

National Sports Medicine Institute, Leesburg, VA USA

National Sports Medicine Institute, Leesburg, VA USA

Cleveland Clinic, Cleveland, OH USA

The Ohio State University, Columbus, OH USA

University of North Carolina Medical Center, Chapel Hill, NC USA

Methodist Sports Medicine, Indianapolis, IN USA

Mayo Clinic Rochester, MN USA

University of California, San Francisco, CA USA

Methodist Sports Medicine Center, Indianapolis, IN USA

Hospital for Special Surgery, New York, NY USA

Washington University in St. Louis, St. Louis, MO USA

Knoxville Orthopaedic Clinic, Knoxville, TN USA

David Geffen School of Medicine at UCLA, Los Angeles, CA USA

University of Colorado Denver School of Medicine, Denver, CO USA 
University of British Columbia, New Westminster, BC Canada

University of Michigan, Ann Arbor, MI USA

Connecticut Children's Medical Center, Hartford, CT USA

Littleton Regional Healthcare, Littleton, NH USA

Warren Alpert Medical School, Brown University, Providence, RI USA

Cleveland Clinic, Cleveland, OH USA

Orthopaedic Associates of Aspen \& Glenwood, Aspen, CO USA

Beth Israel Deaconess Medical Center, Boston, MA USA

State University of New York at Buffalo, Buffalo, NY USA

Methodist Sports Medicine, Indianapolis, IN USA

University of Michigan, Ann Arbor, MI USA

Intermountain Orthopaedics, Boise, ID USA

NYU Hospital for Joint Diseases, New York, NY USA

Robert Larner College of Medicine, University of Vermont, Burlington, VT USA

Washington University in St. Louis, St. Louis, MO USA

University of North Carolina Medical Center, Chapel Hill, NC USA

Keller Army Community Hospital, United States Military Academy, West Point, NY USA

University of North Carolina Medical Center, Chapel Hill, NC USA

Albany Medical Center, Albany, NY USA

Inland Orthopaedic Surgery and Sports Medicine Clinic, Pullman, WA USA

University of Colorado Denver School of Medicine, Denver, CO USA

Royal Columbian Hospital, New Westminster, BC Canada

St. Mary's Audrain, Mexico, MO USA

Cleveland Clinic, Euclid, OH USA

University of Colorado Denver School of Medicine, Denver, CO USA

University of lowa Hospitals and Clinics, lowa City, IA USA

\section{Acknowledgments}

This study received funding from the AOSSM, Smith \& Nephew, National Football League Charities, and Musculoskeletal Transplant Foundation. This project was partially funded by grant No. 5R01-AR060846 from the National Institutes of Health/National Institute of Arthritis and Musculoskeletal and Skin Diseases. 


\section{References}

1. Ahlden M, Samuelsson K, Sernert N, Forssblad M, Karlsson J, Kartus J. The Swedish National Anterior Cruciate Ligament Register: A Report on Baseline Variables and Outcomes of Surgery for Almost 18,000 Patients. Am J Sports Med. 2012; 40(10):2230-2235. [PubMed: 22962296]

2. Ahn JH, Lee YS, Ha HC. Comparison of Revision Surgery with Primary Anterior Cruciate Ligament Reconstruction and Outcome of Revision Surgery between Different Graft Materials. Am J Sports Med. 2008; 36(10):1889-1895. [PubMed: 18490470]

3. Andriolo L, Filardo G, Kon E, et al. Revision Anterior Cruciate Ligament Reconstruction: Clinical Outcome and Evidence for Return to Sport. Knee Surg Sports Traumatol Arthrosc. 2015; 23(10): 2825-2845. [PubMed: 26202138]

4. Bjorkman P, Sandelin J, Harilainen A. A Randomized Prospective Controlled Study with 5-Year Follow-up of Cross-Pin Femoral Fixation Versus Metal Interference Screw Fixation in Anterior Cruciate Ligament Reconstruction. Knee Surg Sports Traumatol Arthrosc. 2015; 23(8):2353-2359. [PubMed: 24832696]

5. Bottoni CR, Smith EL, Shaha J, et al. Autograft Versus Allograft Anterior Cruciate Ligament Reconstruction: A Prospective, Randomized Clinical Study with a Minimum 10-Year Follow-Up. Am J Sports Med. 2015; 43(10):2501-2509. [PubMed: 26311445]

6. Brophy RH, Haas AK, Huston LJ, Nwosu SK, MARS Group. Wright RW. Association of Meniscal Status, Lower Extremity Alignment, and Body Mass Index with Chondrosis at Revision Anterior Cruciate Ligament Reconstruction. Am J Sports Med. 2015

7. De Wall M, Scholes CJ, Patel S, Coolican MR, Parker DA. Tibial Fixation in Anterior Cruciate Ligament Reconstruction: A Prospective Randomized Study Comparing Metal Interference Screw and Staples with a Centrally Placed Polyethylene Screw and Sheath. Am J Sports Med. 2011; 39(9): 1858-1864. [PubMed: 21622815]

8. Filbay SR, Ackerman IN, Russell TG, Macri EM, Crossley KM. Health-Related Quality of Life after Anterior Cruciate Ligament Reconstruction: A Systematic Review. Am J Sports Med. 2014; 42(5):1247-1255. [PubMed: 24318609]

9. George MS, Dunn WR, Spindler KP. Current Concepts Review: Revision Anterior Cruciate Ligament Reconstruction. Am J Sports Med. 2006; 34(12):2026-2037. [PubMed: 17092921]

10. Griffith TB, Allen BJ, Levy BA, Stuart MJ, Dahm DL. Outcomes of Repeat Revision Anterior Cruciate Ligament Reconstruction. Am J Sports Med. 2013; 41(6):1296-1301. [PubMed: 23605223]

11. MARS Group. Factors Influencing Graft Choice in Revision Anterior Cruciate Ligament Reconstruction in the MARS Group. Journal of Knee Surgery. 2015 epub ahead of print.

12. Group M, Group M. Effect of Graft Choice on the Outcome of Revision Anterior Cruciate Ligament Reconstruction in the Multicenter ACL Revision Study (MARS) Cohort. Am J Sports Med. 2014; 42(10):2301-2310. [PubMed: 25274353]

13. Group M, Wright RW, Huston LJ, et al. Descriptive Epidemiology of the Multicenter ACL Revision (MARS) Cohort. Am J Sports Med. 2010; 38(10):1979-1986. [PubMed: 20889962]

14. Hettrich CM, Dunn WR, Reinke EK, Group M, Spindler KP. The Rate of Subsequent Surgery and Predictors after Anterior Cruciate Ligament Reconstruction: Two- and 6-Year Follow-up Results from a Multicenter Cohort. Am J Sports Med. 2013; 41(7):1534-1540. [PubMed: 23722056]

15. Kvist J, Kartus J, Karlsson J, Forssblad M. Results from the Swedish National Anterior Cruciate Ligament Register. Arthroscopy. 2014; 30(7):803-810. [PubMed: 24746404]

16. Lansdown DA, Allen C, Zaid M, et al. A Comprehensive in Vivo Kinematic, Quantitative Mri and Functional Evaluation Following Acl Reconstruction - a Comparison between Mini-Two Incision and Anteromedial Portal Femoral Tunnel Drilling. Knee. 2014

17. Mascarenhas R, Saltzman BM, Sayegh ET, et al. Bioabsorbable Versus Metallic Interference Screws in Anterior Cruciate Ligament Reconstruction: A Systematic Review of Overlapping MetaAnalyses. Arthroscopy. 2015; 31(3):561-568. [PubMed: 25557919]

18. Matava MJ, Arciero RA, Baumgarten KM, et al. Multirater Agreement of the Causes of Anterior Cruciate Ligament Reconstruction Failure: A Radiographic and Video Analysis of the MARS Cohort. Am J Sports Med. 2015; 43(2):310-319. [PubMed: 25537942] 
19. Noh JH, Roh YH, Yang BG, Yi SR, Lee SY. Femoral Tunnel Position on Conventional Magnetic Resonance Imaging after Anterior Cruciate Ligament Reconstruction in Young Men: Transtibial Technique Versus Anteromedial Portal Technique. Arthroscopy. 2013; 29(5):882-890. [PubMed: 23538044]

20. Salmon LJ, Pinczewski LA, Russell VJ, Refshauge K. Revision Anterior Cruciate Ligament Reconstruction with Hamstring Tendon Autograft: 5- to 9-Year Follow-Up. Am J Sports Med. 2006; 34(10):1604-1614. [PubMed: 16685086]

21. Wang H, Fleischli JE, Zheng NN. Transtibial Versus Anteromedial Portal Technique in SingleBundle Anterior Cruciate Ligament Reconstruction: Outcomes of Knee Joint Kinematics During Walking. Am J Sports Med. 2013; 41(8):1847-1856. [PubMed: 23752955]

22. Wegrzyn J, Chouteau J, Philippot R, Fessy MH, Moyen B. Repeat Revision of Anterior Cruciate Ligament Reconstruction: A Retrospective Review of Management and Outcome of 10 Patients with an Average 3-Year Follow-Up. Am J Sports Med. 2009; 37(4):776-785. [PubMed: 19336620]

23. Wright R, Spindler K, Huston L, et al. Revision Acl Reconstruction Outcomes: Moon Cohort. J Knee Surg. 2011; 24(4):289-294. [PubMed: 22303759]

24. Wright RW. Osteoarthritis Classification Scales: Interobserver Reliability and Arthroscopic Correlation. J Bone Joint Surg Am. 2014; 96(14):1145-1151. [PubMed: 25031368]

25. Wright RW, Dunn WR, Amendola A, et al. Anterior Cruciate Ligament Revision Reconstruction: Two-Year Results from the Moon Cohort. J Knee Surg. 2007; 20(4):308-311. [PubMed: 17993075]

26. Wright RW, Gill CS, Chen L, et al. Outcome of Revision Anterior Cruciate Ligament Reconstruction: A Systematic Review. J Bone Joint Surg Am. 2012; 94(6):531-536. [PubMed: 22438002]

27. Wright RW, Huston LJ, Spindler KP, et al. Descriptive Epidemiology of the Multicenter ACL Revision Study (MARS) Cohort. Am J Sports Med. 2010; 38(10):1979-1986. [PubMed: 20889962] 


\section{Table 1}

\section{Baseline Characteristics of Cohort}

\begin{tabular}{lr}
\hline \multicolumn{2}{l}{ N $(r)$} \\
\hline Gender & \\
$\bullet$ Males & $697(58 \%)$ \\
$\bullet$ Females & $508(42 \%)$ \\
Age (years) & 202635 \\
BMI & 22.625 .128 .5 \\
Baseline Activity Level (Marx) & 41116 \\
\hline
\end{tabular}

PREVIOUS SURGICAL INFORMATION

Time since last ACL reconstruction (years)

1.43 .48 .3

Revision number

$\cdot 1$

$1055(88 \%)$

- 2

$125(10 \%)$

- 3 or more

$25(2 \%)$

Surgeon's opinion of failure

- Traumatic

$405(34 \%)$

- Technical

$265(22 \%)$

- Biologic/other

$135(11 \%)$

- Combination

$398(33 \%)$

Cause of technical failure (Surgeon opinion)

- Tunnel malposition

$532(45 \%)$

- Other

$76(6 \%)$

- Combination

$114(10 \%)$

- None

$452(39 \%)$

Surgeon's revision his/her own failure

- No

$859(72 \%)$

- Yes

$341(28 \%)$

Prior surgical technique

- One-incision

$975(81 \%)$

- Two-incision

$203(17 \%)$

- Open Arthrotomy

$22(2 \%)$

Technique of prior femoral tunnel

- Single tunnel

$1167(98 \%)$

- Double tunnel

Previous femoral fixation

- Interference screw

$721(60 \%)$

- Endobutton

$205(17 \%)$

- Cross pin

$149(12 \%)$

- Other

$101(8 \%)$ 


\begin{tabular}{lr}
\hline & $\mathbf{N}(\%)$ \\
\hline$\cdot$ Combination & $25(2 \%)$ \\
Prior femoral tunnel aperture position 1 & \\
$\cdot$ - Ideal & $386(33 \%)$ \\
$\cdot$ Ideal (both position + size), but enlarged tunnels & $28(2 \%)$ \\
$\cdot$ Compromised (position) & $689(58 \%)$ \\
$\cdot$ Compromised (size) & $20(2 \%)$ \\
$\cdot$ Compromised (position + size) & $60(5 \%)$ \\
Prior tibial fixation & \\
$\cdot$ - Interference screw & $857(71 \%)$ \\
$\cdot$ Other & $241(20 \%)$ \\
$\cdot$ Combination & $101(8 \%)$
\end{tabular}

Prior tibial tunnel aperture position 1

- Ideal

- Ideal (both position + size), but enlarged tunnels

- Compromised (position)

- Compromised (size)

- Compromised (position + size)
$721(60 \%)$

$72(6 \%)$

$338(28 \%)$

$35(3 \%)$

$27(2 \%)$

\section{CURRENT SURGICAL INFORMATION}

$\begin{array}{lr}\text { Surgical exposure/technique } & \\ \text { • Anteromedial portal } & 556(46 \%) \\ \text { • Transtibial } & 426(36 \%) \\ \text { • } 2 \text { Incision } & 211(18 \%) \\ \text { • Open Arthrotomy } & 6(1 \%) \\ \text { Notchplasty } & \\ \text { • No } & 277(23 \%) \\ \text { • Yes } & 927(77 \%) \\ \text { Femoral tunnel aperture position } & \\ \text { • Optimum position } & 324(27 \%) \\ \text { • Same tunnel - but compromised position } & 23(2 \%) \\ \text { • Blended new/old tunnel } & 220(18 \%) \\ \text { • Entirely new tunnel } & 590(49 \%) \\ \text { - Added a } 2^{\text {nd }} \text { tunnel } & 45(4 \%) \\ \text { Femoral tunnel bone graft } & \\ \text { • None } & \\ \text { • Staged (prior) } & 1082(90 \%) \\ \text { - Yes (current) } & 87(7 \%) \\ \text { Femoral fixation } & 32(3 \%) \\ \text { • Interference screw (metal) } & \\ \text { • Interference screw (bioabsorbable) } & \\ \text { • Suture + button/endobutton } & 522(43 \%) \\ & 154(13 \%) \\ & 251(21 \%)\end{array}$




\begin{tabular}{|c|c|}
\hline & $\mathbf{N}(\%)$ \\
\hline - Cross pin & $144(12 \%)$ \\
\hline • Other & $55(5 \%)$ \\
\hline - Combination & $76(6 \%)$ \\
\hline \multicolumn{2}{|l|}{ Tibial tunnel aperture position } \\
\hline - Optimum position & $692(58 \%)$ \\
\hline - Same tunnel - but compromised position & $23(2 \%)$ \\
\hline - Blended new tunnel & $248(21 \%)$ \\
\hline • Entirely new tunnel & $199(17 \%)$ \\
\hline - Added a $2^{\text {nd }}$ tunnel & $41(3 \%)$ \\
\hline \multicolumn{2}{|l|}{ Tibial tunnel bone graft } \\
\hline • None & $1076(89 \%)$ \\
\hline - Staged (prior) & $93(8 \%)$ \\
\hline - Yes (current) & $34(3 \%)$ \\
\hline \multicolumn{2}{|l|}{ Tibial fixation } \\
\hline - Interference screw (metal) & $386(32 \%)$ \\
\hline • Interference screw (bioabsorbable) & $297(25 \%)$ \\
\hline - Interference screw + suture & $41(3 \%)$ \\
\hline - Intrafix & $107(9 \%)$ \\
\hline - Other & $124(10 \%)$ \\
\hline - Combination & $247(21 \%)$ \\
\hline \multicolumn{2}{|l|}{ Graft } \\
\hline - Autograft - BTB & $336(28 \%)$ \\
\hline - Autograft - soft tissue & $244(20 \%)$ \\
\hline - Allograft - BTB & $287(24 \%)$ \\
\hline - Allograft - soft tissue & $298(25 \%)$ \\
\hline - Other (ie. autograft +allograft) & $39(3 \%)$ \\
\hline \multicolumn{2}{|l|}{ Biologic enhancement } \\
\hline • No & $1103(92 \%)$ \\
\hline • Yes & $97(8 \%)$ \\
\hline $\begin{array}{l}\text { Knee position at the time of graft fixation (degrees } \\
\text { of flexion) }\end{array}$ & 01020 \\
\hline $\begin{array}{l}\text { Knee position at the time of graft fixation (degrees } \\
\text { of hyperextension) }\end{array}$ & 000 \\
\hline Surgeon experience (years) & 81318 \\
\hline
\end{tabular}

Key: a b crepresents the lower quartile $\mathrm{a}$, the median $\mathrm{b}$, and the upper quartile $\mathrm{c}$ for continuous variables.

${ }^{1}$ All tunnel determinations for position and size are individual surgeons' determinations. BTB = bone-patellar tendon-bone 


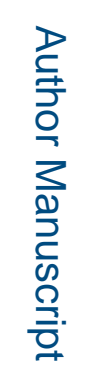

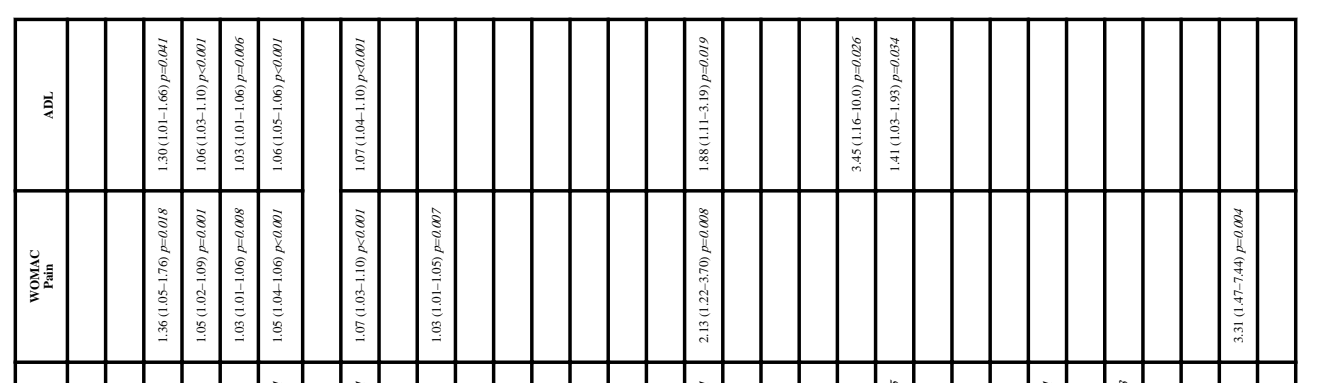

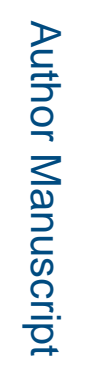

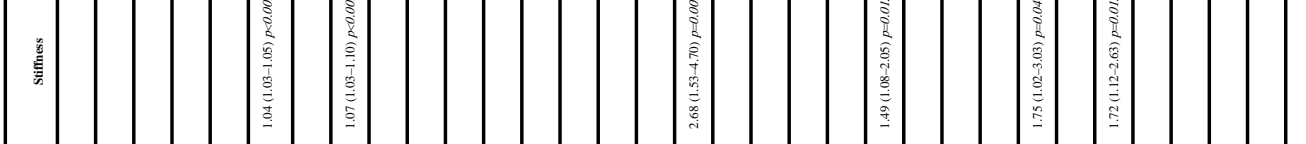

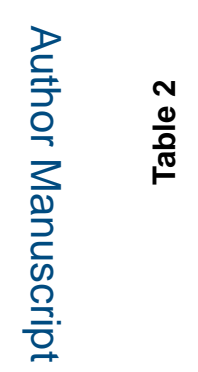

?
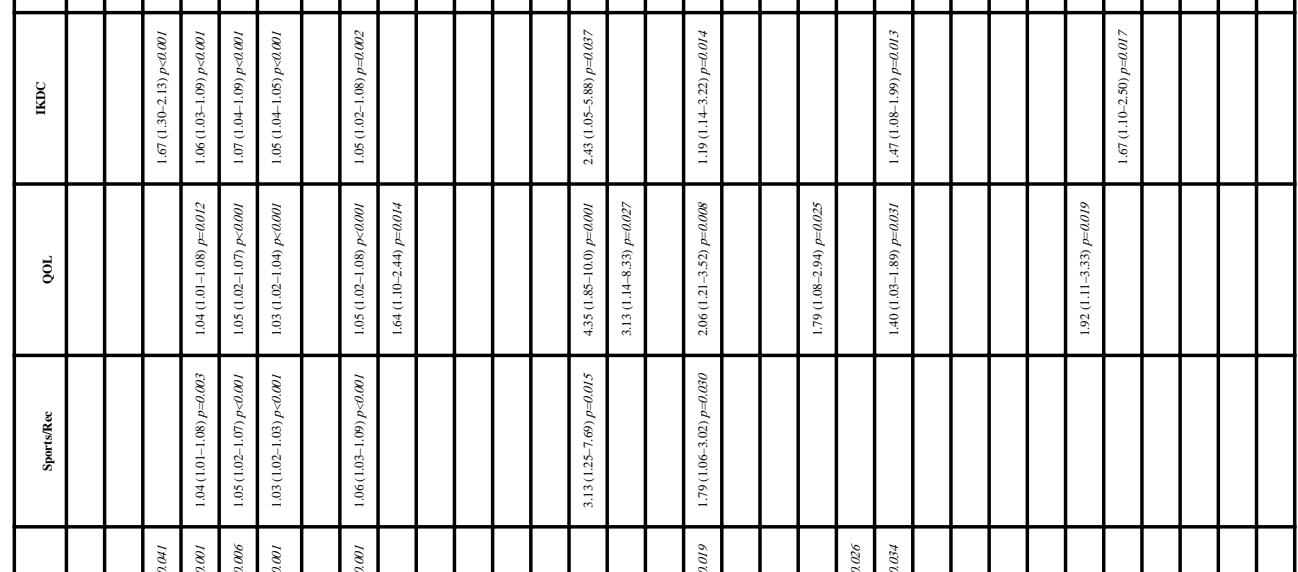

影产
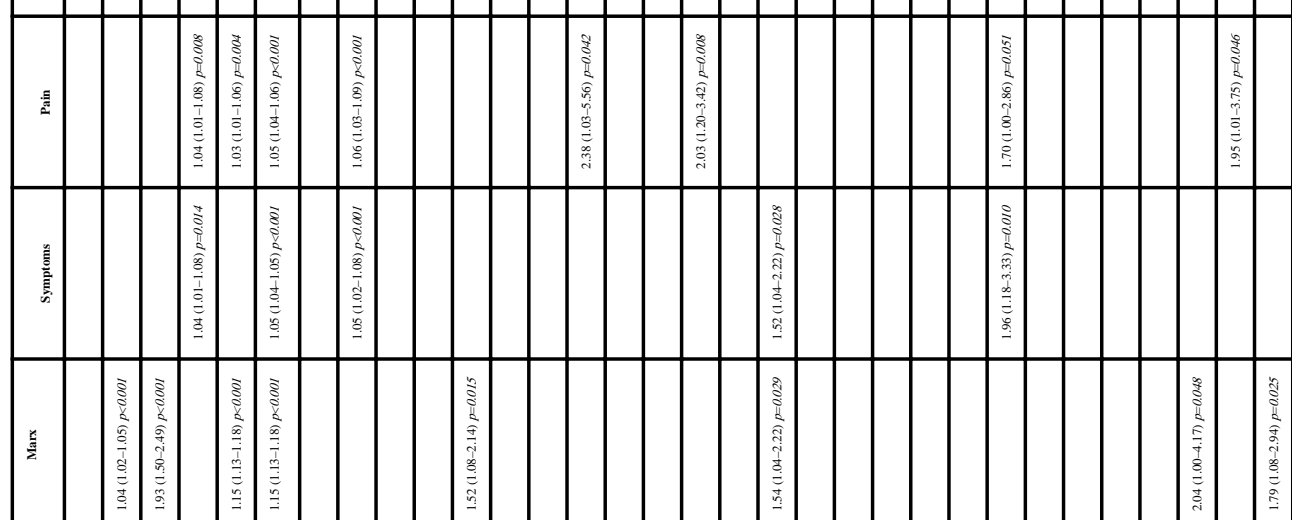

흔

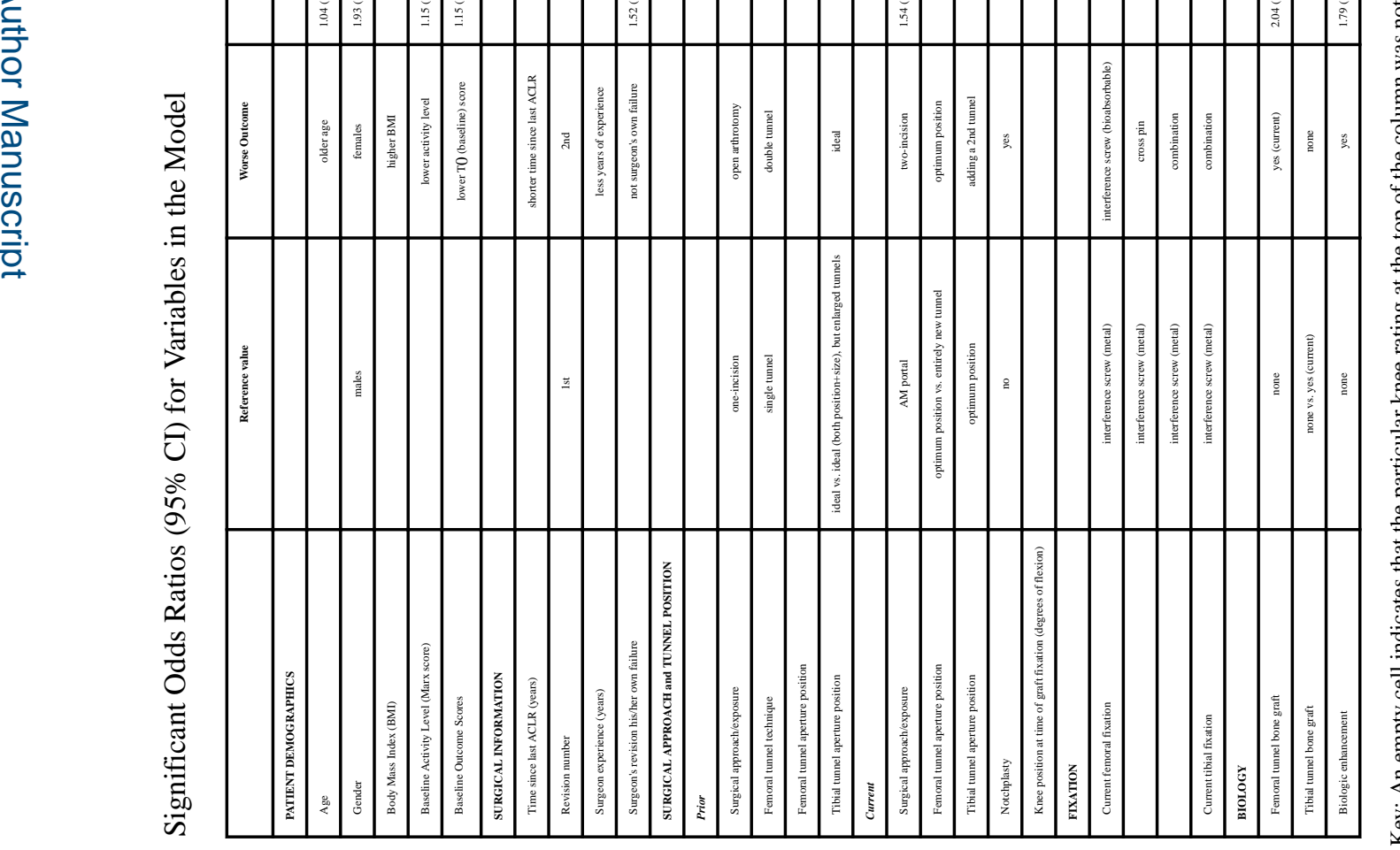

Am J Sports Med. Author manuscript; available in PMC 2018 September 01. 